\title{
Investigation on phase change materials for use in refrigerated transport equipment
}

\author{
Xiao-yan $\mathrm{Li}^{1, \mathrm{a}^{*}}$, Tian-yang $\mathrm{Li}^{1, \mathrm{~b}}$, Liu Yang ${ }^{1, \mathrm{c}}$ \\ ${ }^{1}$ School of Energy and Building Engineering, Harbin University of Commerce, No. 1 Xuehai Street, \\ Songbei District Harbin, Heilongjiang, 150028, China \\ amylxy6168@sina.com, Ity194521@qq.com, ${ }^{\mathrm{c}} 1658135111 @ q q . c o m$
}

Keywords: Cold storage; Refrigerated transport equipment; Phase change material; Thermal performance.

Abstract. This paper presents the preparation for phase change materials(PCM) with different phase change temperature for use in refrigerated transport equipment. The optimal preparation condition of HSD-1 as the PCM was confirmed. The thermo-physical properties of the modified HSD-1 were investigated by cooling curve method and differential scanning calorimeter (DSC) method. The effect of nucleating agent and thickener were deeply researched. The results indicate that the phase change temperature of HSD- 1 is $4.2^{\circ} \mathrm{C}$ and the latent heat is $318.1 \mathrm{~kJ} / \mathrm{kg}$. The modified HSD- 1 has good heat stability. With the injection of $0.0567 \mathrm{~g}$ of PS as the nucleating agent into the HSD-1, the degree of super-cooling decreases by $5^{\circ} \mathrm{C}$. By adding $\mathrm{SJ}$ as the thickener, the temperature of the system is stable and no phase separation is observed. These results show that the modified HSD-1 can be used in refrigerated transport equipment.

\section{Introduction}

With the rapid development of economy, demand on food consumption is much higher than before and technologies related to food cold chain are drawing attention. According to statistics, more than one hundred million tons of food need to be refrigerated transported every year [1]. However, the refrigerated transport technological level is low in China. By the restriction of equipment, form and other factors, the cost of refrigerated transport is high [2]. So the research and development on the refrigerated transport equipment to reduce the cost is necessary. A new mode of transportation which is the combination of phase change cold storage technology and refrigerated transport has become a new issue under discussion $[3,4,5]$. The key technology of phase change cold storage is the selection of phase change material which should have proper phase change temperature fitted for refrigerated transport equipment, high latent heat value, non-poisonous, green and stable after a few charging and discharging cycles $[6,7,8]$.

In this paper, we study the preparation of inorganic liquid phase change material HSD-1 which can be used in refrigerated transport equipment. The eutectic point and latent heat of the binary mixture HSD-1 as the PCM was confirmed by cooling curve method and DSC curves method. The effect of nucleating agent and thickener on crystallization and phase separation of HSD-1 was also investigated experimentally.

\section{Experimental equipments}

Figure 1 shows the schematic diagram of the test system which is used to monitor the cooling curves of PCM consisting of thermostatic water bath, insulation, outer and inner tubes, thermocouple wires and a data acquisition system. The thermal properties of the PCM were measured with a differential scanning calorimeter (DSC) [9]. Before the tests, the temperature and sensitivity calibration of the DSC were investigated. 


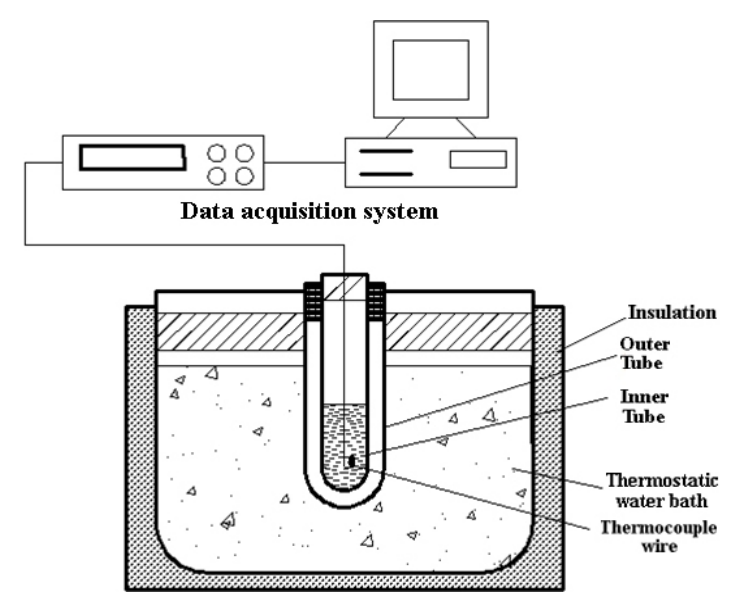

Fig. 1 Schematic diagram of the test system

\section{Thermal performance analysis}

Cooling curve method. Cooling curve method was applied to analyze the thermal performance of the PCMs. By searching the thermophysical parameters, inorganic binary mixture HSD-1 was chosen as the PCMs and investigated. Figure 2 shows the cooling curve for HSD-1.(a) is the cooling curve for HSD- 1 with $15 \%$ of eutectic concentration and (b) is the cooling curve for HSD-1 with $21 \%$ of eutectic concentration.

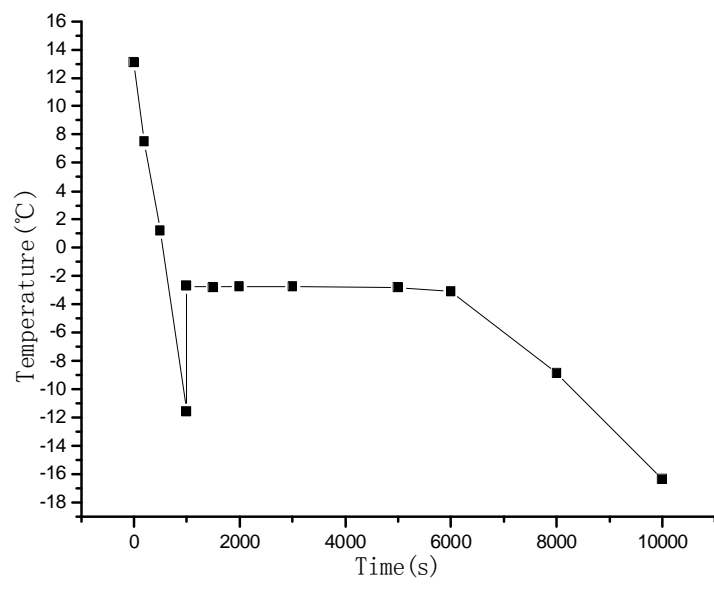

(a)

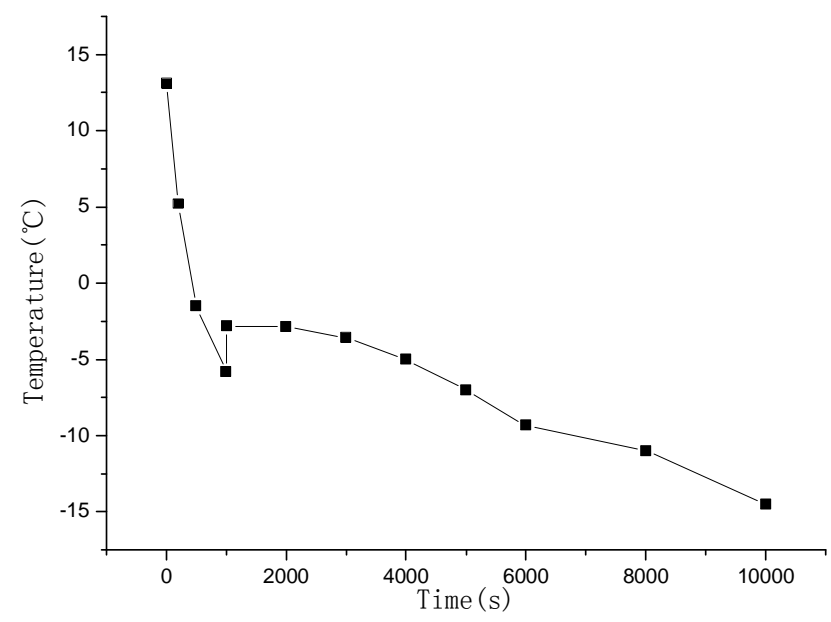

(b)

Fig. 2 Cooling curve for HSD-1

By analyzing the curves, the phase change temperatures and super-cooling temperatures of HSD-1 can be concluded as shown in Table 1.

Table 1 Phase change temperatures and degree of super-cooling of HSD-1

\begin{tabular}{ccc}
\hline Eutectic concentration $(\%)$ & Phase change temperature $\left({ }^{\circ} \mathrm{C}\right)$ & Degree of super-cooling $\left({ }^{\circ} \mathrm{C}\right)$ \\
\hline 15 & -2.7 & 6.8 \\
21 & -2.8 & 3 \\
\hline
\end{tabular}

Table 1 shows that HSD- 1 with $15 \%$ and $21 \%$ of eutectic concentration nearly have the same phase change temperature. The degree of super-cooling is $6.8^{\circ} \mathrm{C}$ for the $\mathrm{PCM}$ with $15 \%$ of eutectic concentration and $3{ }^{\circ} \mathrm{C}$ for the PCM with $15 \%$ of eutectic concentration. However, compared Figure 2 (a) to (b), it can be concluded that the temperature of HSD-1 with $15 \%$ of eutectic concentration decreased to $-9.5^{\circ} \mathrm{C}$ at about $17 \mathrm{~min}$ since the experiment started and increased to $-2.7^{\circ} \mathrm{C}$ rapidly when the super cooling occurred at this period. Then the temperature almost kept unchanged until the experiment time turned to 100min when the temperature started to decrease and the duration that the temperature kept constant is much longer than that of HSD-1 with the eutectic concentration of $21 \%$, 
so HSD-1 with $15 \%$ in eutectic concentration has larger latent heat. Therefore HSD- 1 with the eutectic concentration of $15 \%$ is selected for further study as the optimal preparation condition.

DSC method. To investigate the latent heat of HSD-1, DSC test was undertaken. Figure 3 shows the DSC curve for the melting process of HSD-1. Before the tests, the temperature and sensitivity calibration of the DSC were investigated.

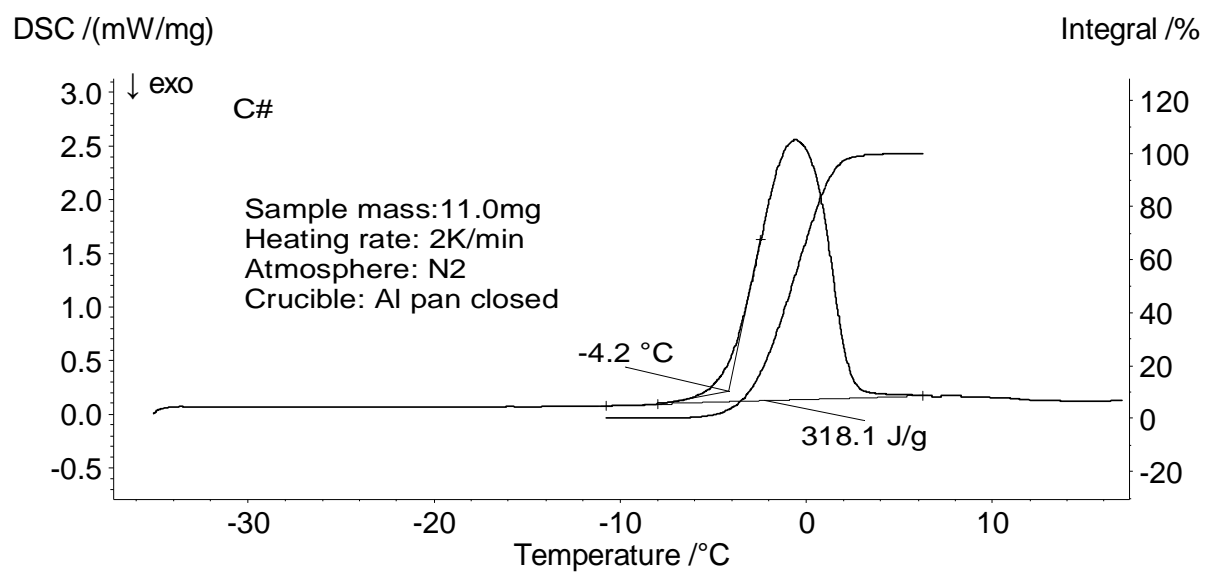

Fig. 3 DSC curve for HSD-1 in melting process

It can be seen from Figure 3, HSD-1 has a melting point of $-4.2^{\circ} \mathrm{C}$, and a latent heat of $318.1 \mathrm{~kJ} / \mathrm{kg}$. The melting point is considered to be the peak temperature, and the latent heat is obtained by integrating the peak range. The DSC output curve becomes flat when a phase change does not occur, and a remarkable variation from the baseline in the output value of the DSC test is found when a phase change occurs.

Thermal stability. The thermal stability of HSD- 1 was studied by repeat tests. After 50 times charging and discharging cycles, the latent heat of HSD-1 increases to 320.1 J/g and the phase change temperature decreases to $-4.3^{\circ} \mathrm{C}$. Compared that to the values of the first test, the latent heat and phase change temperature changed a little. So the thermal stability of HSD-1 is good. HSD-1 can be used as PCM for refrigerated transport equipment.

Effect of nucleating agent on crystallization. The phase change temperature of HSD- 1 is $-4.2^{\circ} \mathrm{C}$, but the PCM experiences super-cooling when phase change occurs which will slow down the crystallization process. So nucleating agent was added to decrease the degree of super-cooling [10]. By searching the existing data, PS was selected as the nucleating agent. 0.0567g of PS was weighed and mixed with $0.67 \mathrm{~g}$ of HSD-1 powers in a tube. The mixture was heated and vibrated until complete dissolution. Then the cooling curves were drawn. The comparison between the cooling curves with and without PS added are shown in Figure 4. Figure 4 shows that the degree of super-cooling decreases from $6.8^{\circ} \mathrm{C}$ to $1.8^{\circ} \mathrm{C}$. So the nucleating agent can effectively decrease the super-cooling of PCM.

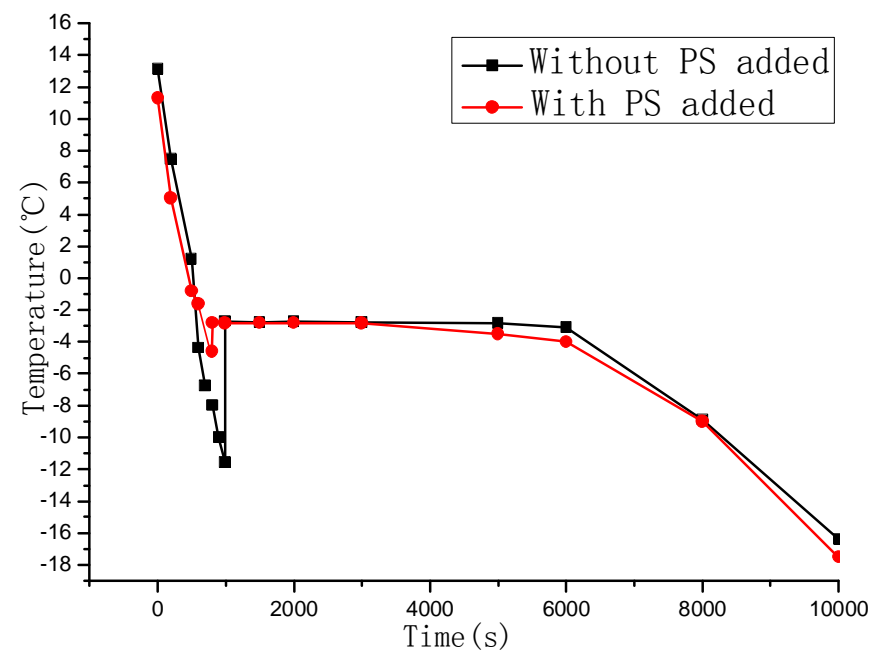

Fig. 4 Cooling curves for HSD-1 with and without PS 
Effect of thickener on phase separation. Phase separation of HSD-1 can be observed because the density difference between the inorganic liquid and solid material which have great effect on the phase change temperature and latent heat. So thickeners were injected to avoid the phase separation [11]. By repeated tests, thickener SJ was selected to decrease the phase separation. With the injection of SJ, the viscosity of HSD-1 increased. During the cooling process, the transparency of the HSD-1 changed and the temperature of the system was stable at the phase change temperature. No phase separation was observed. So the phase separation can be effectively avoided with the thickener added.

\section{Conclusion}

In this paper, we present the research on the preparation of low-temperature inorganic binary mixture HSD-1. The following conclusions can be drawn.

(1) The optimal eutectic concentration of HSD-1 for cold storage is $15 \%$ by analyzing the cooling curves.

(2)HSD-1 has a phase change temperature of $-4.2^{\circ} \mathrm{C}$ and a latent heat of $318.1 \mathrm{~kJ} / \mathrm{kg}$. After 50 times charging and discharging cycles, the variation of latent heat and phase change temperature is small. This shows that HSD-1 has good thermal stability.

(3)The super-cooling and phase separation can be effectively decreased due to the injection of PS as the nucleating agent and SJ as the thickener.

(4)The results of this study indicated that the modified HSD-1 can be used for refrigerated transport equipment.

\section{Acknowledgments}

The authors would like to acknowledge the project (51476049) supported by National Natural Science Foundation of China.

\section{References}

[1] Jiang Shen, Bin Liu. Journal of Refrigeration.06 (2009) 20-25. (In Chinese)

[2] Chenxiao Zheng, Zeqin Liu, Yuan Chang. Journal of Anhui Agri. Sci. 40(33) (2012) 16368-16370, 16461. (In Chinese)

[3] Tan H, Li Y, Tuo H, et al. Energy. 35(5) (2010) 1927-1935.

[4] Ahmed M, Meade O, Medina M A. Energy Conversion and Management. 51(3) (2010) 383-392.

[5] Liu M, Saman W, Bruno F. Applied energy. 92 (2012) 336-342.

[6] Jinping Li, Ruzhu Wang, Kaihua Guo. Energy Technology. 24(3) (2003) 119-121. (In Chinese)

[7] Benlen Z,Jose M,Luisa F. Applied Thermal Engineering. 23(2) (2003) 251-283.

[8] Jinhui Li, Xiaolan Liu, Rongjun Zhang. New Chemical Materials. 34(8) (2006) 18-21. (In Chinese)

[9] S. Cantor. Thermochimica Acta. 26(6) (1978) 39-46.

[10] Ting Li, Xin Pan, Tiejin Ying. Journal of Chinese Institute of Food Science and Technology. 11(4) (2011) 115-122. (In Chinese)

[11] Yudong Liu,Xin Li,Pengfei Hu,Guanghua Hu. International Journal of Refrigeration. 50 (2015) 80-86. 